$\Rightarrow$ INNATE IMMUNITY

\section{Pick a CARD}

DOI:

10.1038/nri1928

9

CARD9 has

helped to

delineate a

novel non-TLR-

dependent

signalling

pathway

The innate immune system of vertebrates detects pathogenic organisms by recognizing the molecular patterns typical of microbial components. Toll-like receptors (TLRs) constitute a major class of pattern-recognition receptors (PRRs), but other PRRs are also important for the recognition of certain pathogens. However, the way in which these PRRs trigger inflammatory pathways is not well understood. A recent study of the poorly characterized protein caspase-recruitment-domain protein 9 (CARD9) has helped to delineate a novel non-TLR-dependent signalling pathway involved in antifungal innate immunity.

CARD9 is structurally related to CARD-MAGUK (membrane-associated guanylate kinase) protein 1 (CARMA1). CARMA1 mediates nuclear factor- $\kappa \mathrm{B}(\mathrm{NF}-\kappa \mathrm{B})$ activation through $\mathrm{BCL}-10$ (B-cell lymphoma 10) and MALT1 (mucosa-associated-lymphoidtissue lymphoma-translocation gene 1) in response to the activation of $\mathrm{T}$ - and $\mathrm{B}$-cell receptors. CARD9 can bind BCL-10 but lacks other regions that are typical of

CARMA-family members

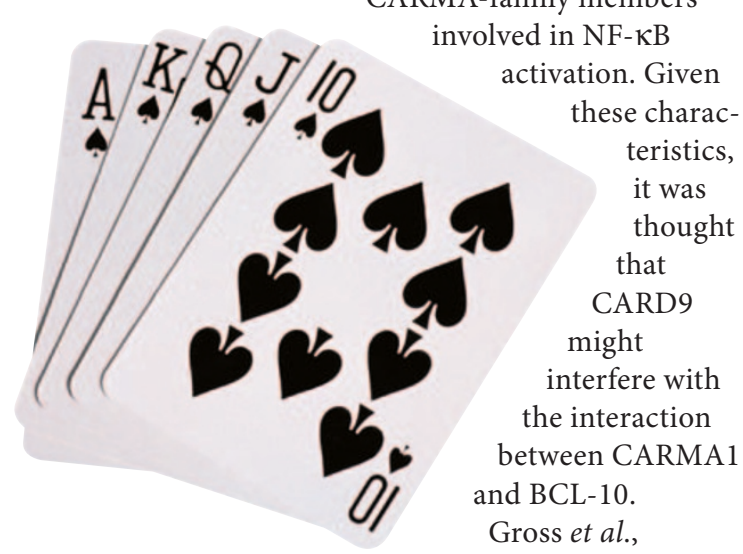

however, found that T- and B-cell function in CARD9-deficient mice resembled that of wild-type mice, and they concluded that CARD9 was probably not involved in receptor signalling through the CARMA1-BCL-10 pathway. When the authors compared the responses of bone-marrow-derived dendritic cells (BMDCs) from CARD9deficient mice and wild-type mice, they discovered that cytokine production induced by zymosan (a cell-wall component of yeast) or by whole fungal cells of Candida albicans was severely impaired in the absence of CARD9. This signalling pathway selectively involved dectin-1, the main mammalian PRR for zymosan, rather than TLR2, which also binds zymosan. Co-expression of CARD9 and BCL-10 showed that these signalling molecules cooperate to induce $\mathrm{NF}-\kappa \mathrm{B}$ activation. Additional studies indicated that BCL-10 and MALT1 are both required for zymosan-induced activation of BMDCs.

The data indicate that CARD9 is an essential link in a newly defined TLR-independent signalling pathway that links zymosan-activated dectin-1 and BCL-10-MALT1mediated activation of NF- $\kappa \mathrm{B}$. CARD9 therefore has a role in innate immunity that is analogous to the role of CARMA1 in adaptive immunity.

Edward Wawrzynczak

ORIGINAL RESEARCH PAPER Gross, O. et al.

Card9 controls a non-TLR signalling pathway for innate anti-fungal immunity. Nature 442, 651-656 (2006) 